\title{
Possibilities of arnica D30 for reducing the procedural pain in the neonatal period.
}

\author{
Penka Petleshkova ${ }^{1 *}$, Maya Krasteva ${ }^{1}$, Iliyana Pacheva ${ }^{2}$, Snezhana Dragusheva ${ }^{3}$, Natalia Vilmosh ${ }^{4}$, \\ Petya Kasnakova ${ }^{5}$, Nikoleta Parahuleva ${ }^{1}$ \\ ${ }^{1}$ Department of Obstetrics and Gynecology, Medical University Plovdiv, Bulgaria \\ ${ }^{2}$ Department of Pediatrics and Medical Genetics, Medical University Plovdiv, Bulgaria \\ ${ }^{3}$ Department of Public Health, Medical University Plovdiv, Bulgaria \\ ${ }^{4}$ Department of Pharmacology and Clinical Pharmacology, Medical University Plovdiv, Bulgaria \\ ${ }^{5}$ Medical college, Medical University Plovdiv, Bulgaria
}

\begin{abstract}
It is known that control of procedural pain in newborns is an ethical act and requires treatment. It includes homeopathic agents used in certain clinical conditions in neonatology. The purpose of this prospective study is to determine the effect of administration of the preparation of Arnica D30 to reduce the severity of procedural pain induced by pricking the heel of the newborn in neonatal screening. Performed in a clinical study of 124 full-term infants aged 72-84 hours divided into two groups: the first group (A) $n=67$ infants, that do not apply anesthesia and a second group (B) $n=57$ infants receiving Arnica D30 in the form of an oral solution three times on the day of the procedure and a time in the range 12-24 hours after the procedure. The results are reported in: $30^{\text {th }}$ sec. and the $5^{\text {th }}$ minute after the puncture of the heel. The same indices were followed in the interval 12-24 hours after the screening. The $3^{\text {th }}$ second after the penetration in the heel in Group A and Group B children did not reveal any credible differences on any of the scale indicators as well as the overall assessment of the severity of procedural pain. Comparison between the two groups of the $5^{\text {th }}$ minute after the procedure found lower estimates in group B (with analgesia). These differences between the groups are also valid for the overall score, which showed lower levels for those who have received an Arnica. The use of Arnica D30 affects the severity of procedural pain in neonates.
\end{abstract}

Keywords: Newborn, Procedural pain, Nonpharmacological methods of treatment, Homeopathy.

Accepted on March 25, 2019

\section{Introduction}

Pain in newborns is complex, individual, subjective, and universal [1]. Research on its markers, assessment and treatment has continued more than 30 years. It is known that in their postnatal development, newborns are often subjected to acute, recurrent and prolonged pain stimuluses that are part of routine care for them [2]. Pain control in the neonatal period has been shown to be beneficial in improving physiological, behavioral and hormonal responses [3]. The increased incidence of painful procedures hides the risk of potential negative consequences in later child development [4]. Independently of the accumulated knowledge in this regards, the management of procedural pain is not yet optimal [5]. Clinical practice uses pharmacological and nonpharmacological methods to control it. In the previous decade, studies focused on the risks of not treating pain and, on the other hand, using more aggressive pharmacological analgesic methods. This requires an in-depth study of the effects of nonpharmacological approaches to controlling and reducing procedural pain [6]. These methods are discussed in the NIDCAP (Neonatal Individualized Developmental Care and Assessment Program), which focuses on reducing stress and pain stimulus during routine procedures in newborns [7].

\section{Purpose}

The aim of this prospective study is to investigate the effect of the use of the preparation of Arnica D30 to reduce the severity of the procedural pain caused by the heel lance of the newborn in neonatal screening.

\section{Material and Methods}

Clinical study of 124 term infants aged 72-84 hours divided into two groups was performed: first group (A) $n=67$ neonates 
without analgesia and second group (B) $n=57$ subjects receiving Arnica D30 under the form of a solution. The average gestational age (g.a.) and mean body weight of newborns of group A are: $38.90 \pm 0.05$ grams and $3243.28 \pm$ 48.40 grams, and those of group B-38.98 \pm 0.06 grams and $3248.33 \pm 56.7$ grams. The two groups did not differ among themselves in terms of g.a. and body weight at birth. Of the total number of patients $50(40.3 \%)$ were born by the normal birth mechanism, $69(55.6 \%)$ by cesarean section, $4(3.2 \%)$ with vacuum extractor and $1(0.80 \%)$ with forceps. All children have normal postnatal adaptation, with no postnatal asphyxia data-Apgar score $1 \mathrm{~min}: 8-10$ and $5 \mathrm{~min}$ : 9-10. The Neonatal Infant Pain Scale (NIPS) is used to assess the severity of procedural pain induced by a heel-stick. Through video surveillance before, during, and after the procedure, the indicators were rated 1 or 0 (only 0.1 or 2 weeping tag) depending on the presence or absence of the feature. Additionally, heart rate and transcutaneous saturation $\left(\mathrm{tSpO}_{2}\right)$ were monitored using a Biocare $\mathrm{iM} / 2014$ monitor. The results were read out at: $30^{\text {th }} \mathrm{sec}$ and at the 5 th minute after the heelstick with tracking and within 12-24 hours after the screening. The Arnica D30 infants from Group B for analgesia were given the following schedule: $3 \times 3$ pills on the day of screening, $1 \times$ 3 pills between 12 and 24 hours per os, as an individual solution with sterile water for a total of $3 \mathrm{ml} / 1 \mathrm{ml}$ on reception. The first is 2 hours before the screening, the second is immediately after it and the rest is 2 hours and 12-24 hours after the procedure.

The obtained results were statistically processed by a descriptive analysis, Chi-squared test (X2 analysis), MannWhitney (U-test) at significance of $\mathrm{p}<0.05$ and presented graphically in tables and figures.

\section{Results}

At the $30^{\text {th }}$ second after the puncture of the heel, between group A and group B no credible differences in any of the scale indicators, as well as in the overall assessment of the severity of procedural pain.

The comparison of all indicators between the two groups in the $5^{\text {th }}$ minute after the procedure found lower values in group B (with analgesia). The differences are statistically reliable only with respect to the following indicators: facial expression $(p=0.002)$, hand movement $(p=0.006)$ and foot movement $(p=0.010)$. These differences between the groups are also valid for the overall score, which showed lower levels in the Arnicatreated patients $(\mathrm{p}=0.025)$ (Table 1$)$

The dynamics of the heart rate (HR) is shown in Figure 1. Comparison between the groups at $30 \mathrm{sec}$ and 5 minutes did not find any credible differences. At the $5^{\text {th }}$ minute, a faster reduction of this physiological pain marker to baseline (pretreatment) was found in the analgesic group (B) compared to the control (A). Traceability in the 12-24 hours interval showed lower variations in the received Arnica.

The measurement of oxygen saturation recorded a better recovery at the $5^{\text {th }}$ minutes in the group receiving Arnica (B).
The statistical significance of the differences in the observed lowest oxygen saturation values in both groups $(p=0.002)$ (77.39\% for $83.3 \%$ respectively for the children in the control group and those receiving the analgesia) was established (Figure 2).

Table 1. Evaluate with NIPS at 5 minutes.

\begin{tabular}{|c|c|c|c|c|c|}
\hline NIPS 5 minutes & Analgesia & $\mathbf{N}$ & Mean & Std. deviation & $\mathbf{p}$ \\
\hline \multirow{2}{*}{ Facial expression } & without analgesia & 67 & 0.73 & 0.449 & \multirow{2}{*}{0.002} \\
\hline & Arnica montana & 57 & 0.40 & 0.495 & \\
\hline \multirow{2}{*}{ Crying } & without analgesia & 67 & 0.90 & 0.837 & \multirow{2}{*}{0.101} \\
\hline & Arnica montana & 57 & 0.58 & 0.885 & \\
\hline \multirow{2}{*}{ Breathing patterns } & without analgesia & 67 & 0.73 & 0.447 & \multirow{2}{*}{0.639} \\
\hline & Arnica montana & 57 & 0.40 & 0.495 & \\
\hline \multirow{2}{*}{ Arms } & without analgesia & 67 & 0.85 & 0.359 & \multirow{2}{*}{0.006} \\
\hline & Arnica montana & 57 & 0.63 & 0.487 & \\
\hline \multirow{2}{*}{ Legs } & without analgesia & 67 & 0.84 & 0.373 & \multirow{2}{*}{0.010} \\
\hline & Arnica montana & 57 & 0.70 & 0.462 & \\
\hline \multirow{2}{*}{ State of arousal } & without analgesia & 67 & 0.33 & 0.475 & \multirow{2}{*}{0.345} \\
\hline & Arnica montana & 57 & 0.29 & 0.458 & \\
\hline \multirow{2}{*}{ Overall assessment } & without analgesia & 67 & 4.37 & 2187 & \multirow{2}{*}{0.025} \\
\hline & Arnica montana & 57 & 2.98 & 2709 & \\
\hline
\end{tabular}

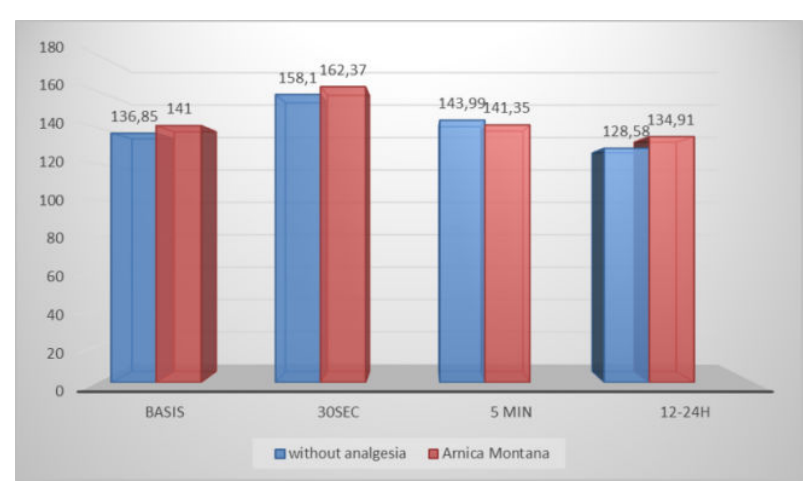

Figure 1. Dynamics of heart rate.

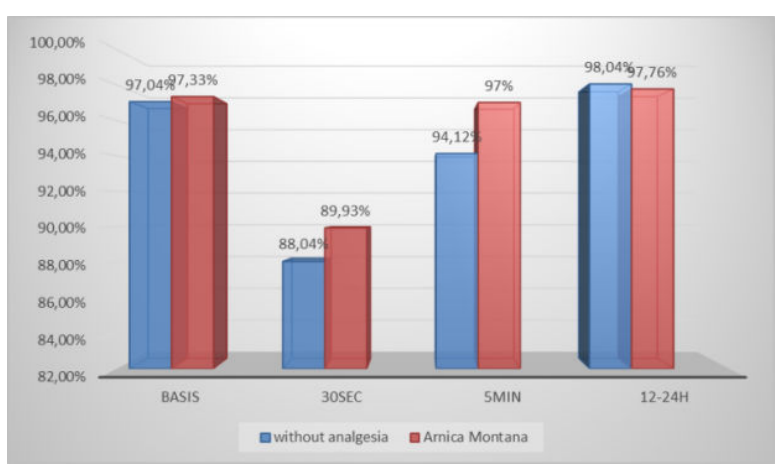

Figure 2. Comparative assessment of oxygen saturation. 


\section{Discussion}

In 1987, Anand and Hickey [4] showed that the nociceptive system was functionally developed in both full-term newborn babies and premature babies. According to studies conducted in relation to analgesia of patients of different ages in given manipulations Anand [4] proves that newborns have increased pain sensitivity compared to other age groups. It has been agreed that their pain manifestations should be monitored by tracking the changes in the pain-behavioral, physiological, hormonal markers on the basis of which the assessment scales are built $[8,9]$.

Progression in neonatal research has shown that newborns suffer pain and that their control has short- and long-term benefits. Varying degrees of neonatal discomfort or pain may occur during routine patient care (e.g., gavage tube placement, bladder catheterization, or physical examination), moderately invasive procedures (e.g., suctioning, phlebotomy, lumbar puncture, heel-stick or peripheral intravenous access), or more invasive procedures (e.g., chest tube placement, circumcision, ophthalmoscopy or central venous access) [8]. This contrasts strongly with the secure and relaxed environment in the womb and coincides with the delicate and critical phase of rapid brain development and stress system programming. Under the impact of frequent and recurrent intensive illness stimuli, the newborn pain system can easily to modify in the wrong way. In healthy infants, studies suggest a change in the threshold of nociception due to pain effects [10], which may lead to secondary effects such as hyperalgesia and allodynia.

Timely and correct assessment of pain is a condition for its management. Various scales are used for this purpose in neonatal practice. One of the established with international consensus is the Neonatal Infant Pain Scale (NIPS). This scale is used for monitoring before and after painful procedures (puncture of heel, etc.) in full-term and premature neonates. It includes a physiological and five behavioral criteria: (1) Face expression; (2) Crying; (3) Breathing patterns; (4) Movement of the hands; (5) Movement of the leg; (6) State of arousal [1]. Pain management of the newborn is a necessity and leads to the elimination or improvement of the markers for it [8].

It is well known that the motor response to healthy term newborn babies in pain after heel-lance is flexion and adduction of the upper and lower limbs associated with grimacing, crying or both. These events are quantifiable. According to Taksande et al. [11], markers such as the brow bulge and the nasolabial furrow appear more often than the crying. This result coincides with our studies where changes in grimacity are observed in most newborns. The pain cry has specific behavioral characteristics and spectrographic properties in healthy full-term neonates. In response to the heel lance, $97 \%$ of follow-up babies showed an increase in behavioral changes [12]. This is confirmed in our results where NIPS scores are higher in the non-analgesic group.

Heart rate and its variability are the most commonly used physiological marker for pain [13]. The magnitude of the changes is related to the intensity and duration of the stimulus and the individual temperament of the infant [14]. South et al. [15] did not show a change in heart rate while Catelin et al. [6] found its reduction in response to pain. Ludington et al [16] and Oberlander et al. [17] detect heart rate increases in nociceptive stimulus. According to Anand, heart rate is increasing following procedures such as: circumcision or puncture of the heel in full-term newborns [14]. Taksande et al. [11] reported the same changes after venipuncture. In the present study, our results coincide with those of Anand and Taksande [2,11] because the heart rate was significantly increased in both groups despite the lack of statistically reliable results.

Physiological markers such as oxygen saturation, blood pressure and respiratory rate have no sensitivity and specificity and cannot be used on their own [13]. According to a study by Pereira et al. [18], oxygen saturation shows a longer reduction after the painful procedure compared to heart rate. Singh et al. [14] and Anand [3] consider that desaturation is a response to painful irritants. The results of this study coincide with those of the abovementioned authors. Hypoxia has been shown to occur even during a routine care procedure. According to Taksande [11], oxygen saturation values can be considered a valid assessment tool about the pain of the newborn. It is possible this stress during the neonatal period to be the reason for subsequent health and psychic complications $[19,20]$.

For the reduction of the pain and its effects on newborns, different non-pharmacological techniques are applied before and during the procedure: flexion position of the limbs, swinging, massage, oral administration of a sweet solution, music therapy, "Kangaroo" care, non-nutritive sucking, contact with mother/speech, caress/homeopathic remedies.

The choice of Arnika Montana is based on its composition and its effects-lactone content (analgesic, anti-inflammatory, antiocclusive effect), phenols (antibacterial action), flavonoids (venous tropism) in the search for additional possibilities for no pharmacologic response to procedural pain in the newborn. is preferred as a non-pharmacological alternative due to the proven in clinical neonatological and pediatric practice in the treatment of traumas with hematomas, open wounds, fractures, headache and other conditions accompanied by varying degrees of pain. [21,22]. In addition, Arnica, administered orally in homeopathic dilutions, showed positive clinical effects in the suppression of post-operative pain, edema and ecchymosis. Local administration of Arnica combined with oral administration has a synergistic effect, reducing postoperative pain. In vitro studies show that the most active components of Arnica, as well as other preparations of the Asteraceae family are helenaline and sesquiterpene lactones-11 $\alpha$, 13-dihydrohelenalin and chamissonolid [23]. One of the earliest evidence of the anti-inflammatory properties of Arnica is reported by Lyss et al. [24]. They find that helenaline inhibits the kappa B (NF- $\mathrm{B})$ transcriptional nuclear factor by altering and stabilizing the NF-kB/kappa B inhibitor (IkappaB) complex in T cells, B cells and epithelial cells and removes kappa gene expression. The quantitative and qualitative content of the sesquiterpene lactones such lactones 
correlates with the proinflammatory cytokines IL-1b and TNF$\alpha$. In addition, treatment with Arnica shows 4.5-fold inhibition of nitric oxide production, a reduction in the levels of inducible cyclooxygenase [23], and reduction of oxidative stress [11].

Puncture of heel is considered the third pain intensity procedure in intensive units [25] and represents $79.2 \%$ of all painful manipulations performed without analgesia [26]. According to the results of this study, the use of Arnica to reduce the severity of procedural pain has a greater impact on behavioral pain markers. This is probably due to their higher sensitivity and lability than the HR and oxygen saturation values. The variance of these indicators gives us a better point of pain [13], which is also evident from our research. We assume that Arnica's lesser influence on vital signs may also be due to the schedule of administration of an analgesic agent whose last intake coincides with the follow-up period of 12-24 hours. Perhaps the weight of the pain stimulus, associated with the particular procedure, also plays an additional role.

In our available literature, we have not found any studies related to the use of homeopathic remedies to control neonatal pain, which limits our ability to compare our results.

Additional studies are needed that can reveal the potential of homeopathic remedies as a non-pharmacological method for reducing procedural pain in the neonatal period.

\section{Conclusion}

1. Arnica D30 administration reduces the severity of procedural pain distinctly at the $5^{\text {th }}$ minute after the puncture of the heel.

2. Arnica pain management does not affect the changes in the parameters: heart rate (HR) and oxygen saturation immediately after the heel prick of the newborn, but it leads to their normalization at the $5^{\text {th }}$ minute and within the period of 12-24 hours.

3. This study complements the spectrum of nonpharmacological methods used in the neonatal period to control procedural pain.

\section{References}

1. Prevention and Management of Procedural Pain in the Neonate: An Update. American Academy of Pediatrics Committee on Fetus and Newborn, Pediatrics 2016.

2. Anand KJS. Prevention and treatment of neonatal pain, Literature review current through, 2017.

3. Anand KJS. Defining pain in newborns: need for a uniform taxonomy?Acta Paediatr 2017; 106:1438-1444.

4. Anand KJS, Hickey PR. Pain and its effects in the human neonate and foetus. N Engl J Med 1987; 317:1327-1347.

5. Burgari R. Homeopathic treatment of newborns and infants-Simillium 2002; 17-40.

6. Catelin C, Tordjman S, Morin V, Oger E, Sizun J. Clinical, physiologic,and biologic impact of environmental and behavioural interventions in neonates during a routine nursing procedure. J Pain 2005; 6:791-797.

7. de Camargo RA, da Costa ED, Catisti R. Effect of the oral administration homeopathic Arnica montana on mitochondrial oxidative stress. Homeopathy 2013; 102:49-53.

8. Hatfield LA. Neonatal pain: What's age got to do with it? Surg Neurol Int 2014; 5:S479-S489.

9. Iannitti T, Medina JCM, Bellavite P, Rottigni V, Palmieri B. Effectiveness and safety of arnica montana in post-surgical setting, pain and inflammation. American Journal of Therapeutics 2016; 23:e184-e197.

10. Krasteva M. Pain in the Neonatal Period I--Physiological aspects, causes, response, diagnosis a long-term effects of neonatal pain. Akush Ginekol 2013; 52:47-53.

11. Taksande A, Vilhekar K, Jain M, ChitreD. Pain response of neonates to venipuncture. Indian J Pediatr 2005; 72:751-753.

12. Krasteva M. Pain in the Neonatal Period-II Nonpharmacological and pharmacological treatment Akush Ginekol 2013; 52:29-37.

13. Kucukoglu S, Kurt S, Aytekin A. The effect of the facilitated tucking position in reducing vaccination-induced pain in newborns. Ital J Pediatr 2015; 41:61.

14. Singh H, Singh D, Soni RK. Comparison of pain response to venepuncture between term and preterm neonates. Indian Pediatr 2000; 37:179-181.

15. South MM, Strauss RA, South AP, Boggess JF, Thorp JM. The use of non-nutritive sucking to decrease the physiologic pain response during neonatal circumcision. a randomized controlled trial. Am J Obstet Gynecol 2005; 193:537-542.

16. Ludington-Hoe SM, Hosseini R, Torowicz DL. Skin-toskin contact(Kangaroo Care) analgesia for preterm infant heel stick. AACN Clin Issues 2005; 16:373-387.

17. Oberlander TF, Eckstein Grunau R, Fitzgerald C, Ellwood AL, Misri S, Rurak D, Riggs KW. Prolonged prenatal psychotropic medication exposure alters neonatal acute pain response. Pediatr Res 2002; 51:443-453.

18. Pereira AL, Guinsburg R, de Almeida MF, Monteiro AC, dos Santos AM, Kopelman BI. Validity of behavioral and physiologic parameters for acute pain assessment of term newborn infants. Sao Paulko Med J 1999; 117:72-80.

19. Mark Shen, Gladys El Chaar. Reducing pain from heel lances in neonates following education on oral sucrose. Int J Clin Pharm 2015; 37:529-536.

20. Anna M, Ekaterina U, Nikoleta P, Mariana P. Prevention of hyaline membrane disease (HMD) in Preterm infants, World journal of pharmacy and pharmaceutical sciences 2016; 5:9-16.

21. Perrone S, Bellieni VC, Negro S, Longini M, Santacroce A, Tataranno ML, Bazzini F, Belvisi E, Picardi A, Proietti F, Iantorno L, Buonocore G. Oxidative stress as a physiological pain response in full-term newborns. Oxidative Medicine and Cellular Longevity 2017; 1-7 
22. Martin P. Arnica and Aconite--homeopathic newborn care. Midwifery Today Int Midwife 2009:30-66.

23. Raeside L. Physiological measures of assessing infant pain: a literature review Br J Nurs, 2011; 20:1370-1376.

24. Lyss G, Schmidt TJ, Merfort I, Pahl HL. Helenalin, an antiinflammatory sesquiterpene lactone from Arnica, selectively inhibits transcription factor NF-kappaB. Biol Chem 1997; 378:951-961.

25. Simons SH,Van Dijk M,Anand KJS Roofthooft D, van Lingen RA, Tibboel D. Do we still hurt newborn babies? A prospective study of procedural pain and analgesia in neonates. Arch Pediatr Adolesc Med 2003; 157:1058-1064.

26. Witt N, Coynor S, Edwards C, Bradshaw H. A Guide to Pain Assessment and Mana gement in the Neonate. Curr Emerg Hosp Med Rep 2016; 4:1-10.

\section{*Correspondence to}

Penka Petleshkova

Department of Obstetrics and Gynecology

Medical University Plovdiv

Plovdiv

Bulgaria 